\title{
MATERNIDADES Y FRONTERAS \\ La fecundidad de las mujeres inmigrantes en España
}

\author{
MOTHERHOOD AND TRANSNATIONAL BORDERS \\ Immigrants' women fertility in Spain
}

\author{
Teresa Castro Martín teresa.castro@cchs.csic.es \\ Instituto de Economía, Geografia y Demografia, Centro de Ciencias Humanas y Sociales, CSIC. Madrid
}

\author{
LUIS RoseRo-BIXBY Irosero@mac.com \\ Universidad de Costa Rica
}

\begin{abstract}
RESUMEN
Este artículo tiene como objetivo examinar los patrones reproductivos de la población inmigrante y evaluar en qué medida difieren de los de la población española, utilizando las biografías migratorias y reproductivas de la Encuesta Nacional de Inmigrantes 2007. Los resultados muestran que la fecundidad de las mujeres inmigrantes difiere poco en su nivel -aunque bastante en su calendario- de la de las españolas, excepto en el caso de las africanas (3,5 hijos por mujer en 2004-2006). Su contribución conjunta al Índice Sintético de Fecundidad de España es de 6,6\%. El artículo también analiza las complejas interrelaciones entre migración y fecundidad, poniendo a prueba algunas de las hipótesis más comunes en la literatura. Las mujeres africanas tienen un pico de elevada fecundidad recién llegadas a España, sobre todo si migraron por razones familiares o ya estaban casadas antes de migrar. El resto de los colectivos muestra una fecundidad excepcionalmente baja en el año previo a la migración y, una vez en España, mantienen las tasas de fecundidad moderadamente bajas que ya tenían en la sociedad de origen. Las mujeres que migraron por razones laborales y las que ya tenían hijos en el país de origen tienen una fecundidad significativamente menor en sus primeros años en España que el resto de mujeres inmigrantes.
\end{abstract}

\section{Palabras Clave}

Encuesta Nacional de Inmigrantes, España, Fecundidad, Inmigración.

\section{Abstract}

This paper aims to examine reproductive patterns of immigrants in Spain and to assess to what extent these patterns differ from those of the native Spanish population, using the migration and reproductive biographies collected in the 2007 National Survey of Immigrants. Our results show that, although fertility timing diverges considerably among groups, immigrants' level of fertility differs little from that of Spaniards, except in the case of North African women (3.5 children per woman in 2004-2006). Immigrants' contribution to Spain's total fertility rate is quite modest: $6.6 \%$. The paper also examines the complex interactions between migration and fertility, testing some of the common hypotheses in the literature. African women have a peak of high fertility shortly after arrival in Spain, particularly if they migrate for family reasons or if they were married before migration. The rest of the immigrant groups have a very low fertility rate in the year prior to migration and, once in Spain, they maintain moderately low fertility rates. Women who migrated for work purposes and women who already had children in the country of origin have significantly lower fertility during their first years in Spain that the rest of immigrant women.

\section{KEYWORDS}

Fertility, Immigrants' fertility, Immigration, Spain, Spanish National Survey of Immigrants. 


\section{INTRODUCCIÓN ${ }^{1}$}

Después de tres décadas de fecundidad por debajo del nivel de reemplazo en España, hay consenso de que ésta permanecerá, a corto, medio y probablemente largo plazo, por debajo de ese umbral de reemplazo (Frejka y Sobotka 2008) y que el envejecimiento progresivo de la población es un proceso inevitable (Mamolo y Scherbov 2009). Sin embargo, la velocidad de este ineludible proceso de envejecimiento, así como el tamaño futuro de la población, puede variar de forma significativa según el nivel de fecundidad sea moderadamente bajo o muy bajo (McDonald 2000). España alcanzó su nivel más bajo de fecundidad en 1998 (1,16 hijos por mujer) y durante el periodo 1993-2002, la fecundidad permaneció por debajo de 1,3 hijos, el umbral que delimita las sociedades de muy baja fecundidad (lowest-low fertility societies) (Kohler, Billari y Ortega 2002). A partir de 1999, sin embargo, se registra cierta recuperación en el índice sintético de fecundidad hasta alcanzar 1,46 en 2008. Asimismo, en este periodo reciente se frena el descenso del número absoluto de nacimientos - un descenso que había sido ininterrumpido desde 1976- y se registra un incremento sostenido de los mismos. Concretamente, el volumen anual de nacimientos pasa de 365.193 en 1998 a 519.779 en 2008, lo que supone un aumento del $42,3 \%$ en un periodo de 10 años. Esta recuperación de la fecundidad no es un fenómeno excepcional en el contexto europeo, pues también se ha observado en el Sur y el Este de Europa. Está por dilucidar el papel que han desempeñado el calendario reproductivo, la inmigración, el contexto económico-laboral y las políticas sociales (Goldstein, Sobotka y Jasilioniene 2009).

Uno de los factores que podrían explicar el ligero aumento reciente de la fecundidad es la ralentización del retraso a la maternidad. La tendencia de retraso a la hora de tener el primer hijo ha sido particularmente rápida en el caso de España: la edad media de las mujeres para tener el primer hijo ha ascendido de 25 años en 1980 a 29,6 en 2008 y actualmente se encuentra entre las más elevadas del mundo. Esta pauta sostenida de retraso provoca que los indicadores transversales de fecundidad infravaloren el nivel real de fecundidad de las cohortes (Ortega y Kohler 2001; Sobotka 2004). Así, aunque el índice sintético de fecundidad ha caído a niveles cercanos a 1,1 hijos por mujer a finales de los años 90 , hasta el momento ninguna cohorte española con fecundidad completa ha tenido una fecundidad inferior a 1,6 hijos por mujer (Sardon 2006). En los últimos años, la tendencia de retraso de la maternidad, aunque se mantiene ascendente, se ha desacelerado, y esto determina que los índices transversales de fecundidad se eleven (Sobotka y Lutz 2009).

\footnotetext{
${ }^{1}$ Agradecimientos: Teresa Castro Martín y Luis Rosero-Bixby son miembros del Grupo de Estudios sobre Población y Sociedad (GEPS) <http://www.geps.es/>. Esta investigación ha contado con financiación de los proyectos del Plan Nacional de I+D+i del Ministerio de Ciencia e Innovación CSO2008-03616/SOCl y CSO2009-11883/SOCl. La participación de Luis Rosero-Bixby contó además con financiación de Wellcome Trust (Grant 072406MF) y del proyecto Fogarty del Centro de Ecología y Demografía de la Universidad de Wisconsin, Madison.
} 
Otro de los factores que subyacen al reciente aumento de la fecundidad son los intensos flujos inmigratorios que ha recibido España en la última década (Arango 2004; Cebolla y González-Férrer 2008). Por una parte, el calendario de la fecundidad de la mayoría de las mujeres inmigrantes es bastante más temprano que el de las mujeres españolas, por lo que contribuyen a desacelerar el retraso a la maternidad para el conjunto de la población. Por otra parte, el nivel de fecundidad de la población inmigrante es en promedio más elevado que el de la población española (Roig y Castro Martín 2007). De ahí el interés que suscita el tema de la fecundidad de los inmigrantes, tanto por sus repercusiones demográficas a corto, medio y largo plazo, como por sus implicaciones económicas y sociales. Sin embargo, es un tema que se enfrenta a múltiples retos de medición, estimación, explicación e interpretación, como trataremos de poner de relieve en este estudio.

Más importante que la contribución de la población inmigrante al nivel agregado de fecundidad del país, es su contribución al número absoluto de nacimientos (Devolder y Treviño 2007). Si en 1996 - primer año en el que se recoge información sobre la nacionalidad de los padres en el registro de nacimientos- sólo el 3,3\% del total de nacimientos era de madre extranjera, esta proporción alcanzó el 20,7\% en 2008 $-23,9 \%$ si contabilizamos los nacimientos de madre o padre extranjero. Es decir, casi uno de cada cuatro nacimientos en España se puede atribuir actualmente a la población inmigrante. ${ }^{2}$ Debe subrayarse que la proporción de nacimientos de padres extranjeros está muy por encima del peso relativo de la población extranjera en el conjunto de la población residente en España (11,4\% en 2008). Esta aparente discrepancia se debe a dos circunstancias: (1) a que en la población inmigrante hay una mayor proporción de personas en edad reproductiva -el $69 \%$ de las mujeres inmigrantes tiene entre $15 \mathrm{y}$ 49 años, comparado con el $48 \%$ de las españolas- y (2) a que estas personas tienen más hijos en promedio, es decir, tienen una fecundidad mayor, aunque este patrón no se puede generalizar a los colectivos procedentes de la Unión Europea o de la Europa Este (Roig y Castro Martín 2007).

El primer objetivo de este artículo es examinar los patrones reproductivos de la población inmigrante y evaluar en qué medida difieren de los de la población española. Al utilizar datos de una sola fuente -la Encuesta Nacional de Inmigrantes 2007- para determinar la fecundidad de las mujeres inmigrantes, el artículo soslaya el principal problema de los análisis convencionales, que combinan datos de dos fuentes de desigual cobertura: el registro de nacimientos y el padrón continuo. Como parte de este primer objetivo, el artículo busca documentar la heterogeneidad existente en pautas reproductivas entre los distintos colectivos de inmigrantes y estimar su contribución al nivel global de fecundidad en España.

\footnotetext{
${ }^{2}$ Esta proporción es relativamente alta si la comparamos con otros países europeos con una tradición de inmigración más larga (Sobotka 2008). No obstante, hay que tener en cuenta que del total de nacimientos de madre extranjera en 2008 (107.475), casi la cuarta parte $(24,7 \%)$ tenía padre español.
} 
Un segundo objetivo es examinar las interrelaciones entre migración y fecundidad, poniendo a prueba algunas de las hipótesis más comunes en la literatura, mediante la comparación de las tasas de fecundidad antes, durante y después de la migración, así como según el tiempo de residencia en España. Como complemento a estos dos objetivos estimaremos, con un modelo multivariable, el efecto de varias características socio-demográficas y de la biografía migratoria en el comportamiento reproductivo de las mujeres inmigrantes, para esclarecer hasta qué punto las diferencias observadas entre los distintos colectivos están vinculadas a su composición socio-demográfica, procesos de selección en origen, motivación de la migración o duración de residencia en España.

\section{EL PROBLEMA DE MEDIR LA FECUNDIDAD DE LA POBLACIÓN INMIGRANTE}

Para medir el nivel de fecundidad de un país o región, es habitual utilizar el Índice Sintético de Fecundidad (ISF), una estimación del número medio de hijos que tendrá una mujer durante su vida fértil. Sin embargo, la forma convencional de calcular este índice presenta varios problemas a la hora de medir la fecundidad de la población inmigrante. En primer lugar, para su cálculo es habitual combinar información de dos fuentes de datos: (a) el número de nacimientos de madre extranjera por edad de la madre del registro de nacimientos, un registro de calidad y con cobertura exhaustiva, y (b) el número de mujeres extranjeras por edad, a través del padrón continuo, un registro cuya calidad es objeto de debate.

La cobertura de la población inmigrante en el padrón continuo ha sido analizada en varios estudios (Devolver y Treviño 2007; González-Enríquez 2009). Por una parte, es muy probable que haya un subregistro de algunos colectivos, como los europeos comunitarios - que no necesitan permiso de residencia-, los inmigrantes recién llegados, y los que no tienen domicilio fijo o temen su deportación. Por otra parte, dado que empadronarse no requiere residencia legal, es imprescindible para obtener acceso a la educación y sanidad pública, y sirve como prueba de residencia para una eventual regularización ordinaria o extraordinaria, la mayoría de los inmigrantes tiene importantes incentivos para empadronarse. Es muy posible, sin embargo, que haya duplicados ${ }^{3} 0$ que la gente que regrese a su país de origen o migre a otro país no se dé de baja en el padrón. Un estudio reciente (Rosero-Bixby, Castro Martín, Reher y Sánchez 2009), que examinó la calidad del padrón de 2005 , validando las cifras de inmigrantes a través de métodos indirectos y fuentes externas, concluyó que la cobertura de la población inmigrante en el padrón es en general buena, pero que hay indicios de sobrerregistro, sobre todo para la población masculina procedente de Rumanía, Marruecos y América Latina. ${ }^{4}$

\footnotetext{
${ }^{3}$ Una persona extranjera que ha cambiado de residencia puede haberse inscrito en varios padrones municipales con diferentes documentos de identidad, como el pasaporte y la tarjeta de residencia, lo que dificulta la eliminación de registros múltiples.

${ }^{4}$ Desde 2006 los extranjeros no comunitarios tienen que renovar su inscripción en el padrón municipal
} 
Este sobrerregistro restaría precisión a las tasas de fecundidad convencionalmente calculadas - subestimando los valores reales-, y sesgaría el análisis de la fecundidad diferencial de la población inmigrante y de su evolución temporal. ${ }^{5}$

Con el fin de soslayar este problema, un estudio previo (Roig y Castro Martín 2007) examinó las diferencias de fecundidad entre mujeres españolas y extranjeras utilizando únicamente los datos del Censo de 2001. A través de un análisis multivariable de la fecundidad reciente, las conclusiones de este estudio sugieren que, una vez controlada la composición socio-demográfica (edad, estado civil, número de hijos previos, educación) de los distintos colectivos inmigrantes, sólo se observan diferencias de fecundidad estadísticamente significativas respecto a las mujeres españolas por parte de las mujeres procedentes del Magreb. Para el resto de los colectivos, las diferencias observadas en los niveles de fecundidad reciente son atribuibles, en gran parte, a diferencias de nivel educativo. Sin embargo, dado que tanto el volumen como la composición de la población inmigrante ha variado de forma significativa desde el momento del Censo, no es obvio que las pautas observadas en 2001 sean válidas para el momento actual.

Un segundo problema del ISF, y más difícil de subsanar, es que no tiene en cuenta que la migración afecta al calendario de la fecundidad. Por ejemplo, para algunos colectivos de inmigrantes se ha documentado que la fecundidad es muy baja antes de la migración, pero que este retraso se compensa una vez efectuada la migración, sobre todo cuando ésta está ligada a la formación de unión (Toulemon 2004). El ISF no tiene en cuenta estas discontinuidades y, por tanto, distorsiona la estimación de la fecundidad de los inmigrantes. Si existe un proceso de aceleración de la fecundidad después de la migración, el ISF tenderá a sobreestimar la fecundidad de los inmigrantes y, si por el contrario, la migración provoca un retraso reproductivo durante un periodo relativamente largo, el ISF tenderá a infraestimar la fecundidad de los mismos (Toulemon 2006).

Los dos procesos anteriormente descritos distorsionan las estimaciones de la fecundidad de la población inmigrante, a veces en direcciones opuestas. En este estudio, trataremos de evitar el primer problema - la combinación de dos fuentes con distinto grado de cobertura y fiabilidad- utilizando datos de una sola fuente, la Encuesta Nacional de Inmigrantes 2007. También intentaremos minimizar el segundo problema, por una parte, calculando tasas de fecundidad para un periodo de 6 años - sin excluir el periodo anterior a la migración - y, por otra parte, estimando el efecto relativo de la edad al migrar y la duración de residencia en España en las tasas de fecundidad. Además, examinaremos la fecundidad completa de la cohorte que ha finalizado su reproducción, indicador que por definición no está sesgado por efectos de calendario.

cada dos años, si no quieren ver borrado su registro. Esta medida probablemente ha reducido la probabilidad de sobrerregistro en los años recientes, pero es necesario un estudio a fondo de su repercusión.

${ }^{5}$ La considerable volatilidad anual de las tasas de fecundidad de la población extranjera en España seguramente refleja no sólo la cambiante distribución de la población extranjera según país de origen, sino también la variable cobertura del padrón en distintos años. 


\section{Perspectivas teóRICAS Y estudios PREVIOS}

En la literatura especializada coexisten múltiples hipótesis para explicar y predecir la compleja interrelación entre migración y fecundidad (Kulu 2005; Milewski 2007). La mayoría de estas hipótesis tratan de detectar los mecanismos que subyacen al cambio de normas y comportamientos reproductivos una vez traspasada una frontera nacional, y pretenden predecir si la migración tiene un impacto reductor o estimulador de la fecundidad. Exponemos a continuación las hipótesis más importantes.

La hipótesis de socialización pone su énfasis en el poder condicionante de las normas y preferencias reproductivas del país de origen, asumiendo la continuidad de las mismas durante todo el curso de vida. Estas normas se interiorizan durante la infancia y juventud, pero también pueden consolidarse a través de los vínculos mantenidos con el país de origen después de la migración. Según esta hipótesis, la primera generación de inmigrantes tiende a mantener las normas, preferencias y patrones reproductivos del país de origen (Abbasi-Shavazi y McDonald 2002), pero las segundas generaciones, socializadas en el país de destino, adoptarán los comportamientos que imperen en la sociedad de acogida.

La hipótesis de asimilación/adaptación es la que hasta el momento ha recibido mayor apoyo empírico en contextos diversos, como Alemania (Mayer \& Riphahn 2000) - Suecia (Andersson 2004). Sostiene que los inmigrantes adaptan sus preferencias y comportamiento reproductivo al del país de destino. ${ }^{6}$ De acuerdo al modelo de asimilación, la integración es un fenómeno complejo que avanza durante todo el curso de vida, y que puede verse afectado por la segregación residencial y social, la amplitud y diversidad de las redes sociales, el acceso al sistema de salud y las barreras laborales, religiosas o lingüísticas. Normalmente es un proceso que ocurre a medio o largo plazo, y la convergencia en preferencias y pautas reproductivas puede llevar más de una generación (Garssen y Nicolaas 2008). Este modelo también implica que la edad a la que se produce la migración es importante, ya que la exposición al contexto normativo y cultural de la sociedad de acogida no tiene el mismo efecto si se produce antes o después de comenzar el periodo reproductivo (Toulemon y Mazuy 2004).

Mientras que la hipótesis de asimilación enfatiza la influencia del cambio de contexto cultural y la adopción gradual de nuevas normas y preferencias reproductivas, la hipótesis de adaptación subraya la influencia del cambio de contexto económico y, por consiguiente, predice que el ajuste reproductivo responde a las oportunidades y barreras económicas presentes en la sociedad de destino. La población inmigrante se enfrenta

\footnotetext{
${ }^{6}$ Aunque la mayoría de los estudios sobre dinámicas familiares de inmigrantes se centran en personas que han migrado a un país con una fecundidad más baja que el país de origen, también puede estudiarse la convergencia del comportamiento reproductivo de los inmigrantes que se mueven a un país con una fecundidad más alta que el país de origen, como es el caso de los inmigrantes rusos en Israel (Nahmias 2004) o los inmigrantes chinos en Estados Unidos (Hwang y Saenz 1997).
} 
normalmente a un mayor coste de vida en el país de destino, pero también a mayores oportunidades educativas y laborales, sobre todo en el caso de las mujeres, lo que aumenta los costes reales y de oportunidad de tener hijos. Dado que estas condiciones económicas están presentes desde la llegada al país de destino, se prevé que el ajuste reproductivo se produzca a corto plazo. Por tanto, las predicciones de las hipótesis de asimilación y adaptación son diferentes. Sin embargo, resulta difícil separar los efectos de adaptación y asimilación porque son procesos que ocurren simultáneamente, y por ello se suelen combinar en una única hipótesis.

Un tercer tipo de hipótesis enfatiza el proceso de selección que supone la migración (Akee 2010). Las personas que emprenden una migración no constituyen un grupo representativo de su sociedad, sino que suelen ser diferentes a las que permanecen en el país de origen, respecto a nivel educativo, tipo de ocupación y situación familiar (estado civil e hijos), así como respecto a otras características más complejas de medir, como el espíritu emprendedor, la apertura a la innovación o las aspiraciones de movilidad social (Feliciano 2005). En el caso de los países en desarrollo, los migrantes suelen tener más capital humano y social que el promedio de la población, y por tanto es presumible que hubieran tenido una fecundidad más baja a la predominante en la sociedad de origen incluso si no hubieran emprendido la migración.

También hay estudios que documentan la interrupción que supone la migración internacional para el proceso reproductivo y de formación familiar. En muchos casos, la fecundidad desciende bruscamente en el periodo inmediatamente anterior y posterior a la migración a raíz de los costes económicos asociados al movimiento migratorio, la separación de los cónyuges que muchas veces conlleva la migración, y las dificultades e incertidumbre económica que rodean al proceso de asentamiento en la sociedad de destino. Esta "interrupción reproductiva" puede ser transitoria y verse compensada posteriormente, una vez lograda la inserción residencial y laboral.

En contra de esta hipótesis, otros estudios han documentado que la migración puede tener un efecto estimulador a corto plazo de la fecundidad. Algunos colectivos inmigrantes tienen una elevada fecundidad poco después de llegar al país de destino, sobre todo si la migración está motivada por la formación de pareja o el reagrupamiento familiar, o si el tiempo de espera antes de la migración ha supuesto un aplazamiento deliberado de la maternidad que quiera compensarse al llegar a la sociedad de destino (Toulemon 2004, Lindstrom y Saucedo 2007). Aunque este fenómeno se ha documentado sólo para algunos colectivos de inmigrantes, pone de relieve que el matrimonio puede constituir una variable intermedia clave entre migración y fecundidad (Hoem y Nedoluzhko 2008).

Algunos estudios recientes también han puesto de manifiesto la influencia del contexto socio-económico y político de la sociedad de acogida. Según Frank y Heuveline (2005), una estratificación social y étnica rígida y una estructura de oportunidades muy desigual para los grupos minoritarios en el país de destino condicionan de forma importante las pautas reproductivas de la población inmigrante, incluso en segundas y terceras generaciones. Las barreras derivadas de la discriminación económica y social pueden impedir la asimilación de valores y comportamientos de la sociedad de destino, 
entre ellos la baja fecundidad. Este es el caso, por ejemplo, de las mujeres mexicanas residentes en Estados Unidos, que tienen una fecundidad más temprana y más elevada que las mujeres en México (Parrado 2011).

El comportamiento reproductivo de las mujeres inmigrantes, sobre todo de aquellas sin permiso de residencia, también puede verse influido por la percepción de un hijo como generador de vínculos y derechos en la sociedad de acogida (Bledsoe 2004; Bledsoe, Houle y Sow 2007). La hipótesis de legitimación asume una interrelación entre migración internacional, status legal y comportamiento demográfico (contraer matrimonio y/o tener un hijo). En el caso de España, se trata de percepciones más que de realidades, ya que la nacionalidad se rige por el criterio de ius sanguinis y no de ius solis, y por tanto, el elemento determinante en el momento de establecer la nacionalidad de los niños nacidos en España es la nacionalidad de los padres y no el lugar de nacimiento (Álvarez Rodríguez 2006). ${ }^{7}$ No obstante, aunque la maternidad no facilite realmente el acceso a la residencia legal, es posible que para muchas mujeres inmigrantes el tener un hijo en el país de acogida tenga un significado de anclaje en la nueva sociedad.

Las hipótesis aquí brevemente expuestas nos pueden conducir a muy distintas conclusiones respecto al impacto a corto plazo y largo plazo de la migración en la fecundidad. La divergencia en pautas reproductivas entre la población inmigrante y nativa dependerá en buena medida de si dominan los efectos de socialización, adaptación, interrupción, selección o legitimación, y de la combinación de efectos predominantes. La evidencia empírica acumulada hasta el momento sugiere que la validez de estas hipótesis depende del país que estemos estudiando. Todas ellas han surgido de la experiencia de un país concreto en el que han sido verificadas, pero no siempre se han podido generalizar a otros contextos. Por ello, es más adecuado hablar de hipótesis complementarias que de hipótesis mutuamente excluyentes, y reconocer que el efecto de la migración en la fecundidad estará condicionado por el origen nacional y étnico de la población inmigrante, por el periodo histórico en el que se produce la migración, y por el contexto socio-económico, la legislación y los mecanismos de integración en la sociedad de acogida.

Con el fin de delimitar nuestros objetivos, especificamos a continuación algunas de las hipótesis específicas que trataremos de contrastar para las mujeres inmigrantes en España:

\footnotetext{
${ }^{7}$ Existen, sin embargo, excepciones por las que el nacido en España puede adquirir la nacionalidad española sin que sus progenitores sean españoles: (a) los nacidos en España de padres extranjeros, si ambos carecieran de nacionalidad o cuando la nacionalidad del país de los padres no se pasa a los hijos automáticamente porque se adquiere por ius soli; (b) los nacidos en España cuya filiación no resulte determinada. Según los datos del registro de nacimientos, que desde 2007 recoge, por primera vez, información sobre la nacionalidad del nacido, sólo el $19,5 \%$ de los nacidos ese año en España con ambos padres extranjeros -o de madre extranjera y no reconocido por el padre- obtuvo la nacionalidad española al nacer.
} 
H.1. Las mujeres inmigrantes que se casaron o tuvieron hijos antes de migrar tienen una fecundidad superior a las que se casaron o tuvieron hijos en España (congruente con la hipótesis de socialización), pero inferior al promedio de la sociedad de origen (congruente con la hipótesis de selección). La convergencia con las pautas de fecundidad de las mujeres españolas se acentúa a medida que aumenta el tiempo de residencia en España (congruente con la hipótesis de adaptación).

H.2. Las mujeres inmigrantes que migraron por razones de formación/reagrupación familiar experimentarán una elevada fecundidad poco después de llegar a España (efecto estimulador), mientras que aquellas que migraron por razones laborales tendrán una tasa de fecundidad muy baja en el periodo inmediatamente previo y posterior a la migración (efecto de interrupción).

\section{DATOS Y MÉTODOS}

Este estudio se basa en la Encuesta Nacional de Inmigrantes (ENI), llevada a cabo por el Instituto Nacional de Estadística (INE) entre Octubre 2006 y Marzo 2007. La muestra de esta encuesta es de 15.465 personas de 16 años o más que han nacido fuera de España y que llevan al menos un año en España o que, en su defecto, tienen intención de residir en España al menos un año (INE 2009; Reher y Requena 2009a). En este análisis utilizamos la información de 7.180 mujeres con edades de 16 a 54 en el momento de la entrevista. ${ }^{8}$ Utilizamos el fichero de microdatos de la encuesta, disponible en la web del INE (http://www.ine.es/prodyser/micro_inmigra.htm), y realizamos el procesamiento y análisis de los datos con el software STATA-10 (StataCorp 2009). Todos los resultados que se presentan están corregidos por las ponderaciones muestrales incluidas en el fichero de la encuesta.

Las ventajas de esta fuente para estudiar las complejas intersecciones entre migración y fecundidad son múltiples. Por una parte, como hemos señalado anteriormente, podemos calcular tasas de fecundidad sin tener que acudir a fuentes externas. Por otra parte, al contar con biografías reproductivas y migratorias retrospectivas, podemos calcular tasas de fecundidad antes, durante y después de la migración, que nos permitirán verificar algunas de las hipótesis presentes en la literatura sobre las interrelaciones entre migración y fecundidad, hipótesis sobre las que hasta ahora sólo podíamos especular para el caso español por falta de datos adecuados. ${ }^{9}$ Otra ventaja de esta fuente, frente

\footnotetext{
${ }^{8}$ Seleccionamos las 7.180 mujeres que tenían de 16 a 54 años en el momento de la entrevista porque éstas tenían de 9 a 45 años al inicio de la observación (Enero de 2000) en el análisis de la fecundidad durante el periodo 2000-2006. Nótese que cada mujer tiene siete años de observación y que su edad para el cálculo de la fecundidad es cambiante en el tiempo.

${ }^{9}$ Las encuestas nacionales de fecundidad no tienen una muestra suficientemente representativa para la población inmigrante por regiones de origen.
} 
a otras, es que recoge datos sobre nacionalidad y lugar de nacimiento. En este análisis hemos optado por definir la población inmigrante como población nacida fuera de España (Malmusi y Jansà 2007), aunque en el análisis multivariable hemos incorporado la adquisición de nacionalidad española como variable independiente.

Existen, sin embargo, algunas limitaciones importantes de esta fuente para el estudio de los patrones reproductivos de la población inmigrante que es necesario explicitar. Una de ellas es que la muestra no incluye un grupo de población nacida en España, que pueda utilizarse como grupo de referencia o contraste. Este inconveniente, sin embargo, puede soslayarse fácilmente utilizando las tasas de fecundidad convencionalmente estimadas con datos del registro de nacimientos y el padrón, ya que las tasas de fecundidad de la población española no están sesgadas por un sobrerregistro padronal. Otra limitación es el pequeño tamaño muestral de algunos grupos de inmigrantes. Por ello, hemos optado por agrupar a las mujeres según su origen en grandes grupos regionales, que describiremos más adelante.

La ENI no recogió la historia completa de nacimientos como suele hacerse en la mayoría de las encuestas demográficas de fecundidad. Sin embargo, sí preguntó el número total de hijos tenidos (y de los fallecidos). Además, para cada hijo co-residente preguntó su edad, el año y el país de nacimiento y, en otra sección del cuestionario, para cada hijo no co-residente preguntó su edad, país de nacimiento y país de residencia actual. Desafortunadamente, para los hijos fallecidos,$^{10}$ la encuesta no preguntó ni la fecha ni el lugar de nacimiento, datos que tuvimos que imputar con procedimientos aleatorios que toman en cuenta el periodo fértil de la mujer y la fecundidad relativa de las distintas edades. Con la información disponible, reconstruimos la biografía reproductiva de las mujeres entrevistadas en la ENI, aunque con el grado de imprecisión propio de no disponer del dato exacto del mes de nacimiento.

A lo largo del estudio, utilizamos dos medidas de la fecundidad:

(1) La fecundidad final de cohorte, medida por el promedio de hijos nacidos vivos tenidos por mujer del grupo de edad de 40 a 54 años ( $N=2.233$ mujeres), es decir, la descendencia final de las cohortes nacidas en 1952-1966.

(2) La fecundidad coyuntural o de periodo, medida por tasas anuales por edad en 2000-2006 y resumida por el índice sintético de fecundidad (ISF), que es la suma de las tasas específicas por edad y que estima la fecundidad final de una cohorte hipotética. En algunos análisis estimamos la fecundidad del periodo completo 2000-2006, sin hacer distinción del lugar de residencia de la mujer. En estos análisis, para mujeres que migraron en el año 2000 o posteriormente, parte de su fecundidad se produjo en el lugar de

\footnotetext{
${ }^{10}$ Los hijos fallecidos representan un $4 \%$ del total, y la mayoría corresponden a mujeres de mayor edad y a un pasado distante, por lo que afectan de forma insignificante las estimaciones realizadas para la fecundidad de las mujeres inmigrantes durante el periodo 2000-2006.
} 
origen. En otros análisis restringimos la medición y el objeto de estudio únicamente a la fecundidad durante la condición de inmigrante, es decir, excluimos la exposición y los nacimientos ocurridos antes del movimiento migratorio. Para tener comparaciones más precisas, restringimos aún más la estimación de la fecundidad al periodo 2004-2006 y únicamente a la ocurrida en España, pues solamente en estos años la ENI registró el mes exacto de la llegada a España. Estas estimaciones para el periodo 2004-2006 de la ENI se comparan con estimaciones derivadas de los datos oficiales del INE de nacimientos (Movimiento Natural de la Población) y de población (padrón continuo).

Todas las tasas de fecundidad las estimamos por edades simples, como cociente entre el número de nacimientos y el número de años-mujer vividos en esa edad. Las curvas de fecundidad por edad simple y región de procedencia las estimamos eliminando con regresión local (comando "lowess" en Stata) el ruido propio del reducido número de observaciones en cada edad. A continuación se describen algunas de las variables utilizadas en los análisis.

\section{Origen de las mujeres inmigrantes}

A diferencia de estudios y datos oficiales que se basan en la nacionalidad de las personas, en este estudio consideramos únicamente el país de nacimiento para definir el origen de las mujeres inmigrantes. El lugar de nacimiento tiene la ventaja analítica de ser una característica fija durante toda la vida. Además, está libre de las ambigüedades de las personas con varias nacionalidades.

Para estudiar el efecto del país de origen de las mujeres inmigrantes en su fecundidad, definimos cinco grandes grupos de continentes o regiones de origen. Una mayor desagregación hubiera sido deseable, pero resultaba en tamaños muestrales pequeños y un reducido poder estadístico para identificar diferencias significativas. Los cinco grandes grupos de origen utilizados son:

1. América Latina y el Caribe (LAC) $(\mathrm{N}=3.318)$. Cinco países representan dos tercios del grupo: Ecuador, Colombia, Argentina, Bolivia y Brasil.

2. África ( $\mathrm{N}=926$ ). Las mujeres procedentes de Marruecos representan el $75 \%$ de este grupo.

3. Europa Occidental y otros países de alto ingreso ${ }^{11}(\mathrm{~N}=1.474)$. El $80 \%$ de este grupo proviene de cinco países: Francia, Portugal, Alemania, Reino Unido e Italia.

4. Europa Oriental $(\mathrm{N}=1.308)$. La mitad de las mujeres en este grupo provienen de Rumanía.

\footnotetext{
${ }^{11}$ Este grupo incluye todos los países europeos englobados en la clasificación de Naciones Unidas en Europa Occidental, Norte y Sur: Alemania, Andorra, Austria, Bélgica, Chipre, Dinamarca, Finlandia, Francia, Grecia, Holanda, Italia, Irlanda, Luxemburgo, Malta, Noruega, Portugal, Reino Unido, Suecia y Suiza. Incluye también otros países de alto ingreso: Canadá, Estados Unidos, Australia, Nueva Zelanda.
} 
5. Asia ( $N=156)$. El reducido tamaño de la muestra de este grupo obliga a extrema cautela en la interpretación de resultados.

\section{Momentos de la trayectoria migratoria}

Definimos cinco momentos o periodos en la vida de las mujeres inmigrantes, relativos al instante del movimiento migratorio:

1. Un año o más previo a la migración

2. El año previo a la migración

3. Los dos años siguientes a la llegada a España

4. De 2 a 5 años cumplidos de residencia en España

5. Seis o más años cumplidos de residencia en España

Para quienes llegaron a España en 2004-2006, la ENI recogió información sobre el mes de llegada. Para quienes llegaron antes del 2004, la ENI recogió información solamente del año de llegada, por lo que tuvimos que asumir que el movimiento ocurrió justo a mitad del año.

\section{Otras características de las mujeres inmigrantes}

Además de la edad de las mujeres inmigrantes, que es una variable que varía en el tiempo, estudiamos dos tipos de características:

1. Las que son fijas en el tiempo (en la edad adulta): cohorte (medida también por la edad a la entrevista), país de nacimiento, nacionalidad española al nacer y nivel educativo.

2. Las correspondientes al momento de la migración: edad al migrar, situación conyugal, número de hijos, situación laboral previa a la migración, si la razón para migrar fue laboral o reagrupación familiar, y si el viaje lo efectuó sola o con familiares.

\section{Metodología}

En una adaptación del modelo de Rodríguez y Cleland (1988), usamos regresión de Poisson para modelar las tasas de fecundidad, ${ }^{12}$ referidas a una curva estándar de fecundidad por edad para cada uno de los cinco grandes grupos de origen de las mujeres inmigrantes (las curvas de fecundidad del periodo 2000-2006 que más adelante se presentan en el gráfico 1). Podemos representar el modelo de la siguiente forma:

\footnotetext{
${ }^{12}$ En realidad, lo que se modela en la regresión de Poisson es el número de eventos (nacimientos) como variable dependiente, pero ejerciendo control en la derecha de la ecuación por el tiempo de exposición.
} 


$$
f_{x j z}=v_{x j} \exp (\beta z)
$$

$f$ es la tasa anual de fecundidad.

$v$ es el patrón estándar de fecundidad por edad y por región de origen.

$x$ es la edad de la mujer (que varía en el tiempo).

$j$ es la región de origen.

$z$ es un vector de características de la mujer inmigrante, incluyendo los distintos momentos respecto a la migración, la educación y las razones para migrar.

$\beta$ es un vector de coeficientes de regresión que miden el efecto de cada característica z sobre la fecundidad, controlando por los efectos de las otras características incluidas en el modelo. El valor exponenciado de estos coeficientes representa la tasa relativa de fecundidad (o razón de tasas) correspondiente a la característica en cuestión y con respecto a un grupo de referencia si se trata de una variable categórica

El modelo, por tanto, mide el grado en que la fecundidad de una persona con características $z$ se aparta del patrón esperado de fecundidad para esa edad y región de origen.

\section{Perfil Socio-demográfico de LA POBlación femenina INMIGRANTE}

Antes de entrar en la materia substantiva del artículo — la fecundidad — conviene familiarizarse con las características del grupo a estudiar: las mujeres inmigrantes de 16 a 54 años de edad entrevistadas en la ENI. Observamos en la tabla 1 que el $46 \%$ de las entrevistadas ha nacido en América Latina o el Caribe, 21\% en Europa Occidental, $18 \%$ en Europa Oriental y $13 \%$ en el continente africano ${ }^{13}$. Una proporción considerable (22\%) tienen nacionalidad española, incluyendo un $7 \%$ que la tiene desde el nacimiento. Estos porcentajes varían significativamente según la región de procedencia. En un extremo están las mujeres nacidas en la Europa Occidental, de las que un $43 \%$ tiene la nacionalidad española $-27 \%$ adquirida al nacer, probablemente por ser hija de emigrantes españoles, ${ }^{14}$ y un $16 \%$ adquirida posteriormente. En el otro extremo están las mujeres nacidas en la Europa Oriental, de las que sólo un 2\% declara tener nacionalidad española.

\footnotetext{
${ }^{13}$ Estos porcentajes no están ponderados. Todos los demás indicadores están ponderados por los pesos muestrales consignados en el fichero de la encuesta.

${ }^{14}$ Hay que señalar que, hasta la Constitución de 1978, las mujeres españolas no podían transmitir la nacionalidad española a sus hijos.
} 
La mayoría de estas mujeres inmigrantes se ha asentado en España hace menos de 6 años (mediana de 5,5 años), aunque el colectivo que procede de Europa Occidental se distingue claramente del resto, con la mitad de las mujeres residiendo en España desde hace casi dos décadas. La edad media de las mujeres inmigrantes en el momento de la migración es de 25,5 años, aunque ésta varía de 20,9 años para las mujeres nacidas en la Europa Occidental a 28,1 años para las nacidas en la Europa Oriental. Este dato nos pone de manifiesto que la experiencia migratoria se gesta y tiene lugar durante una etapa crucial del periodo reproductivo. Para la mayoría de las mujeres, hay una superposición importante entre el proyecto migratorio y el proyecto familiar, y es esta confluencia en la misma etapa vital la que subyace a las complejas interrelaciones entre inmigración y fecundidad.

Se observan diferencias importantes en la distribución de las mujeres por educación según región de origen. Las mujeres nacidas en África y Asia son las que tienen un menor nivel educativo ( $55 \%$ y $37 \%$ respectivamente no han efectuado estudios secundarios). El colectivo con mayor nivel educativo es el de Europa Occidental (un $30 \%$ de las mujeres tienen estudios universitarios). Es importante subrayar también que el nivel educativo de las mujeres nacidas en América Latina es relativamente alto en relación con el nivel educativo medio de la región de origen (Castro Martín y Juárez 1995): el $80 \%$ tiene estudios secundarios, incluyendo $23 \%$ con estudios universitarios. Este rasgo corrobora el efecto de selección que ejerce la migración.

En cuanto a la situación laboral, observamos que el $61 \%$ de las mujeres inmigrantes de 16 a 54 años está trabajando en el momento de la entrevista. Esta cifra es superior a la correspondiente a las mujeres españolas (59\%), según la Encuesta de Población Activa del primer trimestre de 2007, y confirma estudios previos que documentan una mayor tasa de actividad económica femenina entre la población inmigrante que la autóctona (Lorenzo, Suárez y Castro Martín 2007)

La mitad de la población inmigrante está actualmente casada o en unión consensual, aunque los porcentajes son más altos para algunos colectivos, como las mujeres asiáticas $(70 \%)$ y las africanas (66\%). En el momento de la migración, la proporción es obviamente menor (42\%). Se distinguen sobre todo las mujeres nacidas en Europa Occidental, que son también las que migraron más jóvenes, sólo un $28 \%$ formaba parte de una unión conyugal en el momento de migrar ${ }^{15}$.

Con relación a la situación reproductiva previa a la migración, los datos de la tabla 1 nos revelan que el $41 \%$ de las mujeres ya tenía hijos antes de emprender la migración. Esta proporción varía considerablemente entre colectivos, con las proporciones más bajas entre las mujeres nacidas en Europa Occidental (18\%) y en África (29\%) y las más altas entre las mujeres nacidas en Latinoamérica y Europa Oriental —alrededor

\footnotetext{
${ }^{15}$ El colectivo procedente de Europa Occidental presenta, en realidad, una distribución bimodal de la edad a la migración, con una primera alta concentración de migraciones durante la infancia y una segunda en torno a los 25 o 30 años de edad.
} 
Tabla 1.

Características sociodemográficas de las mujeres inmigrantes (16 a 54 años de edad) en la Encuesta Nacional de Inmigrantes 2007

\begin{tabular}{|c|c|c|c|c|c|c|}
\hline Característica & Todas & $\begin{array}{c}\text { América } \\
\text { Latina }\end{array}$ & África & $\begin{array}{c}\text { Europa } \\
\text { Occidental }\end{array}$ & $\begin{array}{l}\text { Europa } \\
\text { Oriental }\end{array}$ & Asia \\
\hline (N) & $(7.182)$ & $(3.318)$ & $(926)$ & $(1.474)$ & $(1.308)$ & $(156)$ \\
\hline \multicolumn{7}{|l|}{ Tiene nacionalidad española } \\
\hline Actual & 22 & 24 & 16 & 43 & 2 & 24 \\
\hline Al nacer & 7 & 3 & 6 & 27 & 0 & 0 \\
\hline Mediana de años en España & 5,5 & 5,5 & 7,5 & 19,5 & 4,5 & 8,5 \\
\hline \multicolumn{7}{|l|}{ Edad media } \\
\hline Actual & 34,5 & 34,1 & 33,9 & 37,7 & 32,5 & 36,4 \\
\hline Al migrar & 25,5 & 26,7 & 23,6 & 20,9 & 28,1 & 24,7 \\
\hline \multicolumn{7}{|l|}{ Educación } \\
\hline Primaria o menos & 24 & 20 & 55 & 17 & 15 & 37 \\
\hline Secundaria & 54 & 57 & 37 & 53 & 64 & 43 \\
\hline Con universidad & 22 & 23 & 8 & 30 & 21 & 20 \\
\hline \multicolumn{7}{|l|}{ Económicamente activas } \\
\hline Actual & 61 & 68 & 34 & 58 & 65 & 59 \\
\hline Al migrar & 46 & 51 & 22 & 41 & 54 & 46 \\
\hline \multicolumn{7}{|l|}{ Con hijos } \\
\hline Actual & 66 & 68 & 70 & 61 & 65 & 72 \\
\hline Al migrar & 41 & 49 & 29 & 18 & 51 & 33 \\
\hline \multicolumn{7}{|c|}{ Con hijos menores de 18 en país de origen } \\
\hline Todas & 10 & 15 & 5 & 1 & 10 & 13 \\
\hline Con <2 años en España & 19 & 28 & 6 & 1 & 15 & $\ldots$ \\
\hline \multicolumn{7}{|l|}{ Tiene pareja } \\
\hline Actual & 50 & 44 & 66 & 50 & 53 & 70 \\
\hline Al migrar & 42 & 41 & 44 & 28 & 54 & 49 \\
\hline \multicolumn{7}{|l|}{ Cómo o por qué migró } \\
\hline Sola, sin familia & 51 & 57 & 39 & 34 & 60 & 54 \\
\hline Por reagrupación & 40 & 33 & 60 & 48 & 36 & 38 \\
\hline Por razones laborales & 46 & 54 & 31 & 16 & 64 & 50 \\
\hline
\end{tabular}

Resultados ponderados por pesos muestrales. Actual $=$ al momento de la entrevista.

Fuente: ENI, 2007 
de la mitad ya eran madres antes de migrar. Esta divergencia entre colectivos también se pone de manifiesto cuando contrastamos la proporción de mujeres que tiene hijos menores de 18 años residiendo en el país de origen en el momento de la entrevista. Las proporciones más elevadas corresponden a las latinoamericanas (15\%) y a las europeas del Este $(10 \%)$. Constatamos también que es entre las mujeres que llevan menos tiempo residiendo en España donde se observa una mayor proporción de familias nucleares transnacionales (el $28 \%$ de las mujeres latinoamericanas que llevan residiendo menos de 2 años en España tienen hijos menores en el país de origen).

También es de interés comparar los distintos colectivos de inmigrantes según la motivación de la migración, ya que ésta va a repercutir en las pautas reproductivas y su calendario. En el conjunto de la población inmigrante tienen mayor peso las razones laborales $(46 \%)$ que la reagrupación familiar $(40 \%)$ como motivo de la migración. Sin embargo, se observan importantes diferencias entre colectivos. Mientras que el $54 \%$ de las mujeres latinoamericanas declaran que la migración tuvo un motivo laboral, sólo el 16\% de las mujeres africanas —en su mayoría marroquíes—-mencionan razones laborales.

\section{LA FECUNDIDAD DE LAS MUJERES INMIGRANTES}

\section{La fecundidad final de cohorte}

El número de hijos tenidos por las mujeres de 40-54 años (cohortes nacidas en 19521966) indica su descendencia final, ya que éstas han completado —o casi completadosu periodo reproductivo. Este indicador, a diferencia del ISF, no se ve distorsionado por cambios en el calendario reproductivo.

Los resultados de la tabla 2 nos muestran que la fecundidad final de las mujeres inmigrantes (2,02 hijos) es $15 \%$ superior a la de las españolas (1,76 hijos) nacidas en 1960 , aunque podemos calificar la diferencia de moderada. El colectivo que más se aleja del nivel de descendencia final de las españolas son las mujeres africanas (3,13 hijos). Solamente la descendencia final de las mujeres nacidas en la Europa Occidental (1,5 hijos) es inferior a la de las españolas.

Esta tabla también nos muestra que una proporción importante de la descendencia final de estas cohortes se produjo en el país de origen: las mujeres de 40-54 años nacidas en el extranjero tenían como media 1,35 hijos -el $66 \%$ de su descendencia finalantes de migrar a España. Existe, sin embargo, una importante heterogeneidad según la región de procedencia: mientras que el $92 \%$ de la descendencia final tuvo lugar en el país de origen para las mujeres de Europa Oriental y el $77 \%$ en el caso de las mujeres latinoamericanas, la proporción correspondiente para las mujeres nacidas en Europa Occidental es de sólo el 32\%. Gran parte de estas diferencias se pueden explicar por la edad media a la que migraron estas cohortes: 40,7 años en el caso de las mujeres nacidas en Europa Oriental frente a 26 años en el caso de las mujeres nacidas en Europa Occidental. 
Tabla 2.

Fecundidad final de cohorte. Inmigrantes de edades

40-54 años (cohortes nacidas en 1952-1966)

\begin{tabular}{|c|c|c|c|c|c|}
\hline \multirow{2}{*}{$\begin{array}{l}\text { Región } \\
\text { de Nacimiento }\end{array}$} & \multirow{2}{*}{$\begin{array}{l}\text { Hijos } \\
\text { tenidos }\end{array}$} & \multicolumn{2}{|c|}{ Hijos antes de migrar } & \multirow{2}{*}{$\begin{array}{l}\text { Edad media } \\
\text { al migrar }\end{array}$} & \multirow[b]{2}{*}{$(\mathrm{N})$} \\
\hline & & Número & Porcentaje & & \\
\hline Total inmigrantes & 2,02 & 1,35 & $66 \%$ & 32,4 & $(2.232)$ \\
\hline América Latina & 2,06 & 1,59 & $77 \%$ & 34,7 & (978) \\
\hline África & 3,13 & 1,83 & $58 \%$ & 27,8 & $(277)$ \\
\hline Europa Occidental & 1,50 & 0,49 & $32 \%$ & 26,0 & (628) \\
\hline Europa Oriental & 1,88 & 1,73 & $92 \%$ & 40,7 & (285) \\
\hline Asia & 2,32 & 1,14 & $49 \%$ & 29,1 & (64) \\
\hline \multicolumn{6}{|l|}{ España cohorte:* } \\
\hline 1955 & 1,90 & & & & \\
\hline 1960 & 1,76 & & & & \\
\hline 1965 & 1,62 & & & & \\
\hline
\end{tabular}

Resultados ponderados por pesos muestrales

* Fuente: Sardon, J,-P, (2006), Recent demographic trends in the developed countries, Population-E 61(3): 197-266, Tabla 4.

\section{La fecundidad del periodo 2000-2006}

La descendencia final de las cohortes de inmigrantes nacidas en 1952-1966 nos proporciona información relevante; sin embargo, la mayoría de estas mujeres tuvieron sus hijos en las décadas de los 70 y 80, por lo que sus pautas no se pueden extrapolar a las mujeres inmigrantes que están teniendo sus hijos en la actualidad. Por ello, presentamos a continuación la estimación de la fecundidad coyuntural o del periodo 2000-2006. ${ }^{16}$

El gráfico 1a presenta las tasas de fecundidad específicas por edad y el ISF para las mujeres inmigrantes, basados en los datos de la ENI, junto con las tasas de fecundidad de España calculadas con el método tradicional, combinando datos del MNP y la

\footnotetext{
${ }^{16}$ Nótese que la estimación se refiere al periodo completo 2000-2006, sin importar en qué país residian o tuvieron sus hijos las mujeres inmigrantes. Más adelante presentamos estimaciones de la fecundidad de las mujeres inmigrantes restringida a su residencia en España.
} 
población estimada a mitad del periodo. El ISF del periodo 2000-2006 para el conjunto de mujeres inmigrantes $(1,78)$ es más elevado que el de España a mitad del periodo (1,31 en 2003), pero hay que subrayar que se sitúa por debajo del umbral de reemplazo $(2,1)$. El gráfico 1a muestra también la importante diversidad de pautas de fecundidad que existen dentro de la población inmigrante, en función de la región de origen. La fecundidad más elevada corresponde a las mujeres nacidas en África (3 hijos por mujer), seguida de la de las mujeres nacidas en América Latina (1,7 hijos por mujer). El ISF de las mujeres nacidas en Europa Occidental $(1,4)$ y Europa Oriental $(1,3)$ no difiere substantivamente del de las españolas.

Los diversos colectivos inmigrantes difieren de las mujeres españolas sobre todo en el calendario reproductivo, es decir, en la edad a la que tienen sus hijos. Las mujeres nacidas en América Latina, por ejemplo, muestran un calendario reproductivo mucho más temprano, característico de las sociedades de origen (Rosero-Bixby, Castro Martín y Martín García 2009). Aunque la diferencia en el ISF es relativamente modesta, la distancia en las tasas de fecundidad a edades jóvenes es considerable: la tasa de fecundidad a los 20 años, por ejemplo, es 4 veces mayor entre las mujeres latinoamericanas que entre las españolas (gráfico $1 b$ ). A pesar de tener un ISF equivalente al de las espa-

Gráfico 1.

Fecundidad de las mujeres inmigrantes en 2000-2006 y de España 2003

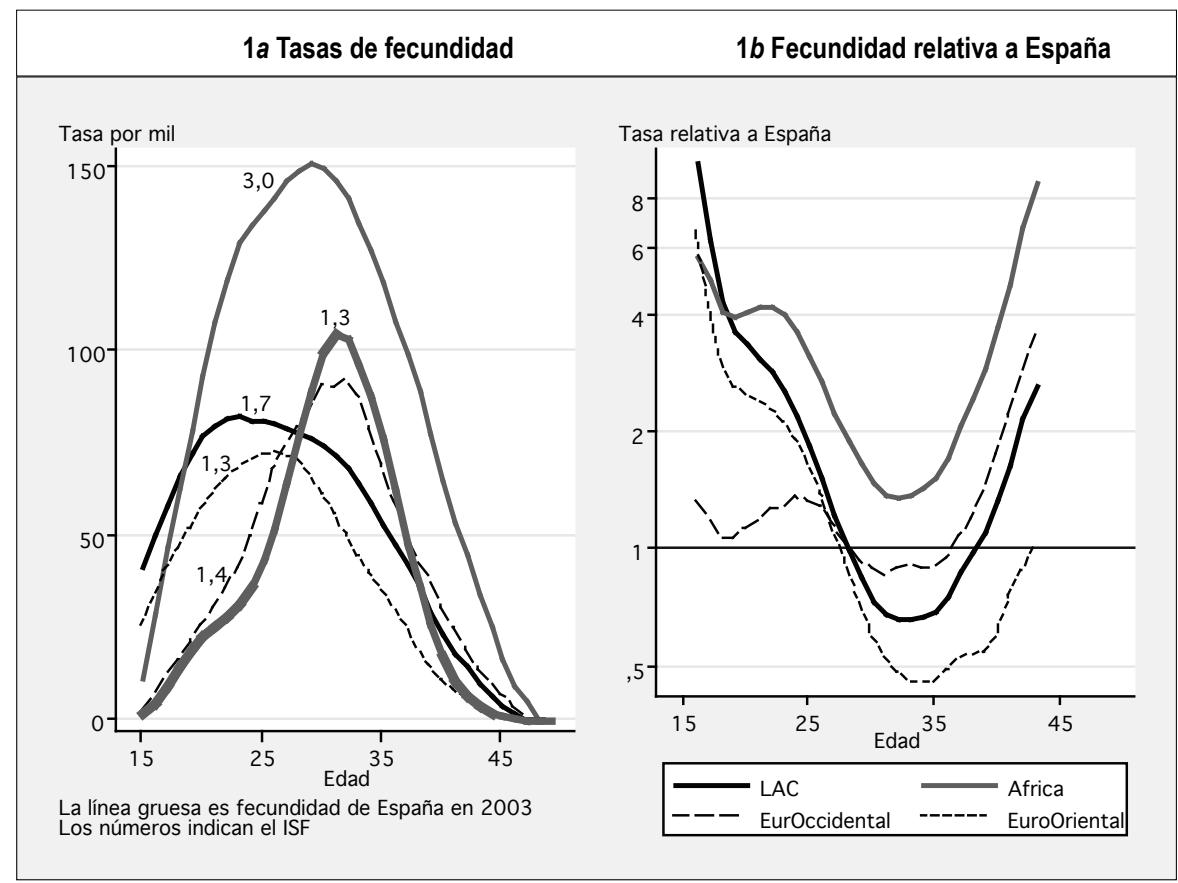


ñolas, las europeas del Este también muestran un calendario reproductivo temprano: su tasa de fecundidad a los 25 años de edad, por ejemplo, es el doble que la de España. A partir de los 27 o 28 años de edad, la situación se invierte y la tasa de fecundidad de España es superior a la de las mujeres inmigrantes, exceptuando las africanas que tienen tasas más altas en todas las edades.

\section{Contribución a la fecundidad en España}

Aunque hemos constatado que todos los colectivos de inmigrantes, a excepción de las mujeres procedentes de Europa Occidental y Oriental, tienen un nivel de fecundidad superior al de las mujeres españolas, estudios anteriores han documentado que su contribución al nivel agregado de fecundidad en España es muy modesta (Roig y Castro Martín 2007). Su protagonismo en la reciente recuperación de la fecundidad es también limitado, aunque no insignificante. Un estudio reciente (Goldstein, Sobotka y Jasilioniene 2009) concluye que el aumento observado en el ISF de España entre 1998 y 2006 se debió fundamentalmente al aumento de las tasas de fecundidad entre las mujeres españolas — de 1,12 a 1,30 hijos. La contribución de las mujeres extranjeras al incremento del ISF durante este periodo fue del $16 \%$. Se han documentado resultados similares en Francia (Héran y Pison 2007), Holanda (Fokkema et al. 2008) y otros países europeos (Sobotka 2008).

En la tabla 3 presentamos nuestro análisis de la contribución de la fecundidad de las mujeres inmigrantes al ISF de España en el periodo 2004-2006. Según este análisis, el incremento del ISF atribuible al conjunto de las mujeres inmigrantes es de 0,082 hijos, es decir, el ISF del periodo 2004-2006 en España hubiera sido de 1,26 hijos por mujer en lugar de 1,34 hijos sin la presencia de las mujeres inmigrantes. Según se desprende de los datos de esta tabla, podemos concluir que, aunque la fecundidad de las mujeres inmigrantes es $56 \%{ }^{17}$ superior a la de las españolas y su impacto en el número de nacimientos -y por consiguiente, en el crecimiento natural de la población- es considerable, su impacto en el ISF es relativamente modesto: sólo lo incrementan un 6,6\%.

Esta tabla también nos permite comparar el ISF estimado con la ENI con el estimado convencionalmente a partir del registro de nacimientos y el padrón. Las dos estimaciones del ISF dan resultados aproximados para el conjunto de todas las mujeres inmigrantes: 1,74 con los registros oficiales y 1,95 con la ENI. Discrepancias mayores se observan cuando comparamos colectivos por región de origen, lo que en parte puede

\footnotetext{
${ }^{17}$ Conviene señalar que el grado en que el ISF de las inmigrantes es superior al de las españolas (56\%) probablemente exagera la distancia real en la fecundidad de estos grupos, ya que el ISF de las mujeres españolas es excesivamente bajo por los efectos de aplazamiento de nacimientos al que hemos aludido en la introducción, mientras que el ISF de las mujeres inmigrantes puede estar exagerado por efectos de recuperación temporal de nacimientos. A este respecto, es posiblemente más realista la comparación de la fecundidad final de cohorte, según la cual las mujeres inmigrantes tendrían solamente $15 \%$ más hijos que las españolas.
} 
deberse simplemente a errores aleatorios de la muestra, que se magnifican a medida que se reduce el número de observaciones. Otra causa de discrepancia se origina en que hemos utilizado el país de nacimiento para definir los inmigrantes en la ENI mientras que los registros oficiales definen a los inmigrantes según su ciudadanía. Esta discrepancia puede magnificarse en colectivos como el de las mujeres nacidas en Europa Occidental, con una elevada proporción de personas con nacionalidad española (que no estarían contabilizadas en los registros oficiales como población extranjera). Y una tercera posibilidad, que se ha examinado en detalle en otro estudio (Rosero-Bixby, Castro Martín, Reher y Sánchez 2009) es que exista un sobrerregistro de la población inmigrante en el padrón continuo que lleve a subestimar su fecundidad en la medida en que el registro nacimientos no sufre de ese problema.

Conviene señalar que la comparación con las estimaciones basadas en el registro de nacimientos y el padrón, así como el análisis del impacto de la inmigración que se presenta en la tabla 3, están restringidos al periodo 2004-2006 y que únicamente se tienen en cuenta los nacimientos y tiempos de exposición en España. Este análisis fue posible hacerlo con mayor precisión que el anterior del periodo 2000-2006, debido a que

Tabla 3.

Indicadores del impacto demográfico de la fecundidad de las mujeres inmigrantes 2004-2006

\begin{tabular}{|c|c|c|c|c|c|c|}
\hline \multirow[b]{2}{*}{ Indicador } & \multicolumn{3}{|c|}{ América } & \multirow{2}{*}{$\begin{array}{c}\text { Europa } \\
\text { Occidental }\end{array}$} & \multirow{2}{*}{$\begin{array}{l}\text { Europa } \\
\text { Este }\end{array}$} & \multirow[b]{2}{*}{ Asia } \\
\hline & Total & Latina & África & & & \\
\hline \multicolumn{7}{|l|}{ ISF } \\
\hline MNP y padrón & 1,74 & 1,43 & 3,53 & 1,23 & 1,54 & 2,43 \\
\hline $\mathrm{ENI}$ & 1,95 & 1,74 & 3,46 & 1,69 & 1,48 & 1,81 \\
\hline \multicolumn{7}{|l|}{ Cocientes del ISF } \\
\hline MNP y padrón /ENI & 0,89 & 0,82 & 1,02 & 0,73 & 1,04 & 1,34 \\
\hline Inmigrantes/ españolas ${ }^{a}$ & 1,56 & 1,39 & 2,77 & 1,36 & 1,19 & 1,45 \\
\hline \multicolumn{7}{|l|}{$\%$ de inmigrantes en: } \\
\hline Nacimientos 2004-2006 & 15,2 & 6,1 & 4,1 & 1,5 & 2,5 & 0,9 \\
\hline Mujeres en edad reproductiva ${ }^{b}$ & 10,6 & 5,2 & 1,4 & 1,6 & 2,0 & 0,5 \\
\hline \multicolumn{7}{|l|}{$\begin{array}{l}\text { Incremento del ISF atribuible } \\
\text { a inmigración }\end{array}$} \\
\hline Nacimientos/mujer & 0,082 & 0,034 & 0,039 & 0,016 & 0,013 & 0,011 \\
\hline$\%$ & 6,6 & 2,7 & 3,1 & 1,2 & 1,1 & 0,9 \\
\hline
\end{tabular}

${ }^{a}$ ISF de mujeres con nacionalidad española $=1,259$.

${ }^{b}$ Mujeres de 15 a 49 años a mitad de 2005 (promedio de padrones municipales a 1 de Enero de 2005 y 2006 ). 


\section{Gráfico 2.}

Tasas de fecundidad por edad de las mujeres inmigrantes y las mujeres de nacionalidad española en 2004-2006



la ENI preguntó a partir del 2004 el mes de llegada a España. El gráfico 2 muestra las curvas de fecundidad de este periodo para el conjunto de mujeres inmigrantes y para las ciudadanas españolas. El gráfico confirma que la mayor fecundidad de las mujeres inmigrantes tiene lugar sobre todo a edades jóvenes. En realidad, de los 31 a los 38 años de edad, son las ciudadanas españolas quienes tienen una mayor fecundidad.

\section{La fecundidad antes y después de la migración}

Varios estudios han señalado que la acomodación del calendario de la fecundidad al calendario de la migración puede sesgar las medidas transversales de la fecundidad, como el ISF. Por ejemplo, si las tasas de fecundidad de la población inmigrante son muy bajas antes de la migración, pero aumentan fuertemente en el periodo inmediatamente posterior a la migración, el ISF de la población inmigrante estaría sobreestimado, y el sesgo sería mayor en aquellas sociedades de destino donde la mayoría de la población inmigrante ha llegado recientemente (Sobotka y Lutz 2009).

Con el modelo de regresión múltiple de Poisson estimamos el efecto de la variable "momento migratorio" en la fecundidad de las mujeres inmigrantes en 2000-2006. El modelo, tal y como fue formulado, controla por la edad de la mujer, o más exactamente, por la fecundidad esperada considerando la edad y el colectivo de origen. Para especificar correctamente el modelo, subdividimos cada observación en segmentos que corresponden a cada edad y momento migratorio. Se estimaron errores estándar robus- 
tos, es decir, tomando en cuenta que los distintos segmentos corresponden a la misma persona. Después de estimar un modelo saturado con 8 variables dummies y 20 interacciones, identificamos un modelo parsimonioso con únicamente efectos significativos. Este modelo confirma que existen diferencias significativas en la fecundidad según el momento migratorio y que este efecto difiere para las mujeres originarias de África y las restantes. No se identificaron diferencias significativas entre los otros colectivos, por ejemplo, entre latinoamericanas y europeas.

El gráfico 3 presenta en forma resumida los resultados del modelo para dos edades arbitrarias: 25 y 35 años. Se representan en él las tasas de fecundidad (y su intervalo de confianza al 95\%) predichas por el modelo según los años antes o después de la migración. Como referencia, el gráfico también representa la fecundidad de España en la edad respectiva. Observamos que para las mujeres que no provienen de África no hay un efecto significativo del momento migratorio en la fecundidad, excepto por una fuerte caída - de cerca del 50\% - en el año previo a la migración. Aparte de esta caída, la fecundidad a los dos, cuatro 0 seis años después de la migración es la misma que la del periodo de un año o más antes de la migración. La fuerte caída de la fecundidad en el año previo a la migración puede interpretarse como un efecto anticipatorio del movimiento migratorio, pero también como un efecto de selección de las personas que migran: es poco probable que las mujeres con un recién nacido emprendan una migración.

El patrón que presentan las mujeres procedentes de África es muy diferente. Las tasas de fecundidad son relativamente bajas antes de la migración - sin que se produzca una caída brusca en el periodo inmediatamente anterior a ésta-, y aumentan notablemente (se multiplican casi por tres) en los dos primeros años después de la llegada a España, para moderarse después (caen un 28\% en el periodo 2-4 años y un $30 \%$ en el periodo de 6 y más años). Este patrón de fecundidad excepcionalmente alta justo después de la migración ha sido documentado para varios países, como Francia (Toulemon 2004) o Suecia (Andersson 2004). En el caso de España, parece que esto sólo ocurre entre las mujeres procedentes de África, que en su mayoría son mujeres marroquíes, para las que la migración está estrechamente vinculada a la formación de pareja o la reagrupación familiar. La relativamente baja fecundidad (para el contexto africano) anterior a la migración de este colectivo podría ser el resultado de una disminución del riesgo de casarse o tener hijos debido a la ausencia del esposo o la pareja, es decir, un paréntesis de espera para llevar a cabo conjuntamente el proyecto de migrar y formar una familia.

De acuerdo con estos resultados, el proceso de convergencia hacia la fecundidad de la sociedad de destino sólo tendría lugar entre las inmigrantes africanas, aunque dadas sus altas tasas originales se requerirán bastantes años - 0 una generación más- para que alcancen la fecundidad española. En el resto de los colectivos inmigrantes, la convergencia no se da por una reducción de la fecundidad una vez integrados en la sociedad de destino, sino por provenir de una sociedad de baja fecundidad, como en el caso de las mujeres nacidas en Europa Occidental y Oriental (congruente con la hipótesis de socialización), o por el proceso de selección ligado a la migración. Este último proceso 


\section{Gráfico 3.}

Fecundidad de las mujeres inmigrantes según el momento migratorio (Tasa predicha para edades 25 y 35 con modelo de regresión de Poisson)

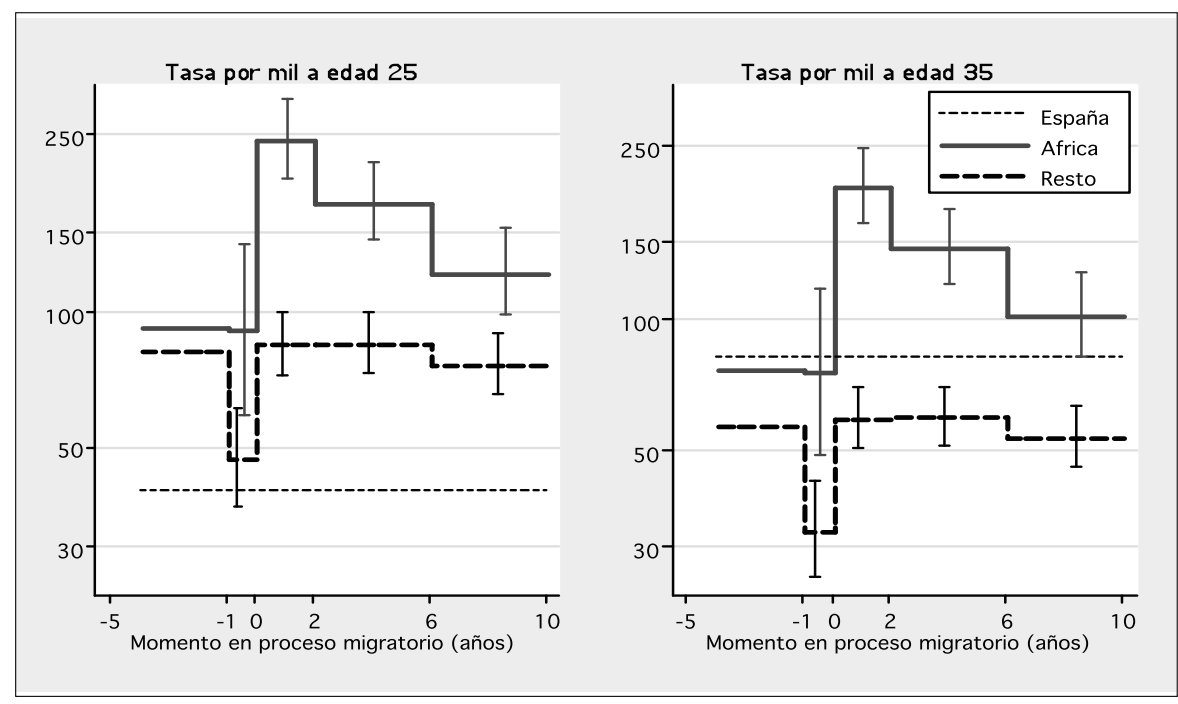

Nota: Las líneas verticales representan los intervalos de confianza.

sería el dominante en el caso de las mujeres latinoamericanas, que por tener un perfil educativo, laboral y familiar diferente al promedio de la sociedad de origen, tienen una fecundidad relativamente baja, no muy superior a la de las europeas y españolas.

\section{Otros determinantes de la fecundidad de las mujeres inmigrantes}

Además de la edad, la región de origen y el momento de la trayectoria migratoria, cuyos efectos hemos analizado en la sección anterior, hay otros muchos factores y características personales que inciden en la fecundidad de las mujeres inmigrantes. Con el fin de explorar la influencia de estos factores, expandimos el modelo anterior de regresión de Poisson, incluyendo variables adicionales, como el nivel educativo, la nacionalidad al nacer, las características previas a la migración (actividad laboral, situación conyugal y reproductiva), la edad en el momento de migrar, la motivación de la migración, y el contexto de la migración (sola o en familia). Esta información, raramente disponible en los registros o encuestas sobre las que se basan la mayoría de los estudios sobre la fecundidad de los inmigrantes en los países europeos, nos permitirá, a su vez, contrastar las hipótesis que hemos formulado anteriormente. Con este objetivo, incluimos variables de interacción entre la duración de residencia en España y los indicadores de haber estado casada o haber tenido hijos antes de migrar y los motivos migratorios laborales 0 de 
reunificación familiar. Analizamos únicamente la etapa post-migratoria del periodo 20002006 para poder incluir en el modelo las características de la mujer en el momento de la migración como variables explicativas fijas en el tiempo. Dado que no se registraban diferencias significativas entre el periodo de 2-5 años y el de 6 y más años después de la migración, estos periodos se unificaron en uno solo. Se estimó un modelo para todas las mujeres inmigrantes y modelos separados para las mujeres nacidas en África y el grupo restante, puesto que, como hemos señalado anteriormente, los efectos del momento respecto a la migración difieren significativamente en estos dos grupos.

La tabla 4 presenta las razones de tasas (RT) de fecundidad estimadas por los coeficientes de regresión del modelo. Por ejemplo, la RT de 0,76 (IC: 0,63-0,92) de quienes migraron siendo menores de 16 años indica que su fecundidad en España es $24 \%$ más baja que el grupo de referencia (las mujeres que tenían de 16 a 29 años al migrar), y este efecto es estadísticamente significativo e independiente de la edad a la que tiene lugar la fecundidad, el colectivo de origen y las otras variables incluidas en el modelo, como el nivel educativo o las razones de la migración.

En contra de lo que esperaríamos, la cohorte, el nivel educativo, la situación laboral previa a la migración y la nacionalidad española al nacer no tienen un efecto estadísticamente significativo en las tasas relativas de fecundidad de las mujeres inmigrantes después de la migración, una vez que se controla por todas las variables incluidas en el modelo. En cambio, la edad al emprender la migración sí tiene efectos significativos importantes: las mujeres que migraron jóvenes (antes de los 16 años) o a una edad madura (después de los 30 años) tienen unas tasas de fecundidad relativamente más bajas que aquellas que migraron en el rango de edad de 16 a 29 años.

La duración de residencia en España (menos de 2 años frente a más de 2 años) presenta efectos complejos, condicionados por otras variables cuyas interacciones se incluyeron en el modelo. En esos dos primeros años, la fecundidad tiende a ser más alta que en el periodo posterior para aquellas mujeres que tenían pareja antes de migrar. Por ejemplo, las mujeres que migraron estando ya casadas o con pareja tienen una fecundidad en España 35\% superior a las que migraron solteras, y sus tasas tienen un incremento adicional del $22 \%$ en los dos primeros años. Encontramos este mismo patrón amplificado entre las mujeres casadas procedentes de África que migraron por reagrupación familiar. Una inmigrante africana que estaba casada antes de migrar y migró por reagrupación familiar tendrá una tasa de fecundidad durante los dos primeros años 3,6 veces $(1,31 \times 1,41 \times 1,31 \times 1,16 \times 1,30=3,65)$ superior a la de una mujer africana que no reúne esas características y lleva más de dos años residiendo en España.

Sin embargo, este patrón de fecundidad relativamente más elevada durante los dos primeros años en España desaparece o se invierte entre las mujeres que ya tenían hijos antes de migrar o que migraron por razones laborales. Las mujeres que migraron después de ser madres tienen una fecundidad en España 27\% inferior a las que migraron antes de tener hijos, y sus tasas tienen una reducción adicional del $22 \%$ en los dos primeros años después de la llegada a España. 
Tabla 4.

Razón de tasas (RT) de fecundidad estimadas con regresión de Poisson según características de las mujeres inmigrantes. Periodo posterior a la migración en 2000-2006

\begin{tabular}{|c|c|c|c|c|c|}
\hline & RT & \multicolumn{2}{|c|}{ (IC 95\%) } & RT África & RT Resto \\
\hline Primaria o menos & 1,09 & $(0,96$ & $1,24)$ & 1,06 & 1,07 \\
\hline Secundaria & 1,00 & & & 1,00 & 1,00 \\
\hline Superior & 0,98 & $(0,86$ & $1,11)$ & 0,94 & 0,99 \\
\hline Nacionalidad española al nacer & 1,02 & $(0,84$ & $1,23)$ & 1,16 & 0,97 \\
\hline Edad actual $35+$ años & 1,05 & $(0,93$ & $1,19)$ & 0,80 & $1,13^{x}$ \\
\hline$<2$ años de llegada & $1,24 \times$ & $(0,99$ & $1,55)$ & 1,31 & 1,20 \\
\hline \multicolumn{6}{|l|}{ Características al migrar } \\
\hline Edad<16 años & 0,76 * & $(0,63$ & $0,92)$ & 0,73 & $0,77^{*}$ \\
\hline Edad 16 a 29 años & 1,00 & & & 1,00 & 1,00 \\
\hline Edad 30 y más años & 0,81 * & $(0,67$ & $0,98)$ & 1,13 & 0,73 * \\
\hline Tenia trabajo & 1,05 & $(0,94$ & $1,18)$ & $1,19 \times$ & 1,05 \\
\hline Migró sola, sin familia & 1,03 & $(0,91$ & $1,16)$ & 1,12 & 0,99 \\
\hline Tenía pareja & $1,35^{\star}$ & $(1,14$ & $1,60)$ & $1,41^{*}$ & 1,34 * \\
\hline$y<2$ años llegada & 1,22 & $(0,93$ & $1,60)$ & 1,31 & 1,11 \\
\hline Tenía Hijos & $0,73^{*}$ & $(0,61$ & $0,88)$ & 0,99 & 0,66 * \\
\hline$y<2$ años llegada & $0,78^{x}$ & $(0,58$ & $1,03)$ & $0,66^{x}$ & 0,85 \\
\hline Migró por razones laborales & 1,05 & $(0,90$ & $1,22)$ & 0,97 & 1,12 \\
\hline$y<2$ años llegada & $0,73^{*}$ & $(0,57$ & $0,94)$ & 0,50 * & 0,80 \\
\hline \multirow{2}{*}{$\begin{array}{l}\text { Migró por reagrupación familiar } \\
\text { y<2 años llegada }\end{array}$} & 1,02 & $(0,88$ & $1,18)$ & 1,16 & 0,98 \\
\hline & 1,15 & $(0,90$ & $1,46)$ & $1,30^{x}$ & 1,02 \\
\hline
\end{tabular}

Notas: Categorias de referencia entre paréntesis. El modelo controla también por edad y región de origen.

* Significativo a $|\mathrm{P}|<0,05$

$\times$ Significativo a $|\mathrm{P}|<0,10$

IC = Intervalo de confianza al $95 \%$ 
En resumen, las interrelaciones entre migración y fecundidad son complejas y los patrones observados difieren significativamente en función de la región de origen, la situación familiar previa a la migración y la motivación de la migración. Los resultados presentados confirman la hipótesis H.2 que habíamos formulado: las mujeres inmigrantes que migraron por razones de formación/reagrupación familiar experimentan una elevada fecundidad poco después de llegar a España (efecto estimulador), mientras que aquellas que migraron por razones laborales tienen una tasa de fecundidad relativamente baja en el periodo inmediatamente previo y posterior a la migración (efecto de interrupción). Sin embargo, hay que puntualizar que sólo encontramos evidencia del efecto estimulador de la migración para las mujeres nacidas en el continente africano.

En cuanto a la primera hipótesis, los resultados obtenidos a través de los diversos análisis realizados, nos obligan a rectificar algunas expectativas y a matizar otras. Por ejemplo, en nuestra hipótesis H.1 inicial habíamos anticipado que las mujeres que se casaron o tuvieron hijos antes de migrar tendrían una fecundidad superior a las que migraron solteras y sin hijos (congruente con la hipótesis de socialización). Sin embargo, los resultados muestran que, aunque las mujeres emparejadas antes de la migración sí tienen una fecundidad más elevada cuando llegan a España, las mujeres que ya han sido madres en el país de origen tienen una fecundidad relativamente más baja en el país de destino. También hemos presentado resultados congruentes con la hipótesis de selección: las mujeres que migran no sólo tienen un nivel educativo superior al promedio de la región de origen, sino que tienen una fecundidad baja antes de emprender el proyecto migratorio. En cuanto al proceso de adaptación de la fecundidad de las mujeres inmigrantes y su convergencia hacia el nivel de las españolas, tenemos que matizar que, aunque habíamos anticipado que este proceso se produjese en todos los colectivos de inmigrantes, en realidad sólo podemos documentarlo para las mujeres procedentes de África. Para el resto, la convergencia no se da por una reducción de la fecundidad una vez integradas en la sociedad de destino, sino por provenir de una sociedad de baja fecundidad, como es el caso de las mujeres nacidas en la Europa del Este, o por el proceso de selección vinculado a la migración —respecto al perfil sociodemográfico y las preferencias reproductivas-, como es el caso de las mujeres latinoamericanas.

\section{Conclusiones}

La inmigración ha provocado una de las más importantes transformaciones socio-demográficas de la sociedad española en los últimos tiempos (Izquierdo y López de Lera 2006; Reher y Requena 2009b). Además del crecimiento poblacional que ha supuesto, la población inmigrante ha contribuido de forma notable al rápido aumento del número de nacimientos - revirtiendo dos décadas de descenso sostenido del volumen de nacidos-, y a ralentizar la tendencia de retraso de la maternidad, así como a atenuar el proceso de envejecimiento demográfico. Sin embargo, en este estudio hemos documentado que la repercusión de la inmigración en el Índice Sintético de Fecundidad de 2004- 
2006 es muy modesta ( 0.08 hijos). Las principales razones de esta pequeña repercusión son que las mujeres inmigrantes sólo representan el $10,6 \%{ }^{18}$ del conjunto de mujeres en edad reproductiva, y que su fecundidad (1,95 hijos), aunque superior a la de las españolas (1,26 hijos), no puede considerarse elevada.

Habría que matizar también que aunque la contribución "directa" de la inmigración al nivel de fecundidad de España es modesta, su contribución "indirecta", aunque mucho más difícil de cuantificar, es trascendental. Dada la escasez de políticas y servicios públicos que ayuden a conciliar las responsabilidades laborales y familiares en España (Navarro 2006), las estrategias de conciliación de madres y padres laboralmente activos suelen sustentarse en la ayuda de las redes familiares - generalmente las abuelas- (Fernández Cordón y Tobío 2005) y en la contratación de ayuda doméstica, que proporcionan mayoritariamente mujeres inmigrantes (Peterson 2007; Tobío y Díaz Gorfinkiel 2007). Esto ha dado origen a las llamadas "cadenas globales de cuidados"19 de dimensiones transnacionales, sin las que la fecundidad de las mujeres españolas en edad reproductiva, mayoritariamente incorporadas al mercado de trabajo, sería probablemente todavía más baja (Martínez Buján 2008).

En este estudio hemos explorado la interrelación entre la migración y el tempo y quantum de la fecundidad. Para ello, hemos documentado la influencia de la situación familiar previa a la migración, de la etapa en el curso de vida en la que se produce la migración y de las motivaciones por las que se emprende la migración en las pautas reproductivas de las mujeres inmigrantes una vez que llegan a España. Esta información es novedosa, ya que hasta ahora no había ninguna fuente que recogiese las biografías migratorias, conyugales y reproductivas de la población inmigrante.

El análisis efectuado también nos ha permitido hacer explícitos los problemas de medición de la fecundidad de la población inmigrante y los posibles sesgos de las mediciones convencionales. Las biografías migratorias y reproductivas retrospectivas de la ENI no sólo han servido para calcular tasas de fecundidad alternativas, sino para poner de manifiesto el reto que supone el diseño de indicadores de fecundidad que tengan en cuenta la compleja interrelación entre migración y fecundidad. Como señala Bledsoe (2004), el periodo reproductivo tiene lugar dentro de un marco temporal específico, pero no necesariamente en un único contexto espacial. Los índices que miden la fecundidad, sin embargo, se calculan convencionalmente asumiendo un lugar o contexto fijo. Según

\footnotetext{
${ }^{18}$ El peso de las mujeres inmigrantes en el conjunto de mujeres en edad reproductiva (15-49) está calculado para 2005, dado que las estimaciones se refieren al periodo 2004-2006. Según datos más recientes (a 1 de enero de 2009), este peso relativo ha aumentado a $15,5 \%$.

${ }^{19}$ El concepto de cadenas globales de cuidados, acuñado por Hochschild (2000), intenta reflejar las interconexiones que en un marco internacional se producen entre unas madres que contratan cuidados para sus hijos en el mercado y otras que los brindan de manera remunerada, teniendo a su vez que encontrar a alguien que las sustituya en el cuidado o supervisión de sus hijos. Las cadenas de cuidado se conforman como cadenas femeninas, reproduciéndose así la división sexual del trabajo a un nivel global (Díaz Gorfinkiel 2008).
} 
esta autora, uno de los principales retos demográficos es vincular tiempo y espacio, analizando no sólo cómo las personas distribuyen sus transiciones vitales en el tiempo, ${ }^{20}$ sino también a través del espacio, aunque traspasen fronteras nacionales.

A este respecto, las ventajas y potencialidades que ofrece la ENI con respecto a otras fuentes son muchas a pesar de que la encuesta no registró detalles importantes de la historia de nacimientos, como el mes en que éstos ocurrieron. No sólo nos ha permitido incorporar al análisis de la fecundidad los hijos nacidos en el periodo previo y posterior a la migración, sino que nos ha brindado la oportunidad de explorar la influencia de la situación de la mujer previa a la migración (situación conyugal, reproductiva, educativa y laboral) en sus pautas reproductivas después de llegar a la sociedad de destino. Gracias a esta detallada información, hemos podido hallar evidencia para algunas de las hipótesis que encontramos en la literatura especializada, como la de selección de las personas que migran en términos de características socio-económicas y preferencias reproductivas - aunque también es plausible que la migración en sí misma constituya una experiencia que transforma la vida de los migrantes en múltiples facetas, incluyendo sus aspiraciones familiares y reproductivas y la renegociación de los roles de género (Wagner 2008).

Sin embargo, para otras hipótesis, hemos llegado a la conclusión de que no pueden generalizarse a todos los colectivos inmigrantes. Por ejemplo, sólo se observa un proceso gradual de adaptación al nivel de fecundidad de la sociedad de destino entre las mujeres africanas, porque en el resto de los colectivos la fecundidad de partida es relativamente baja. Asimismo, la fecundidad en los dos primeros años después de la llegada está fuertemente condicionada por la motivación de la migración. Si la migración tiene como objetivo la formación o reagrupación familiar, se observa una fecundidad excepcionalmente alta tras llegar a España. En cambio, si la migración tiene un carácter laboral, el efecto de interrupción reproductiva es el dominante.

Quedan por supuesto muchos aspectos relevantes de la interrelación entre migración y fecundidad por investigar en el futuro. Sería conveniente, por ejemplo, extender el análisis que hemos realizado aquí basándonos en el comportamiento reproductivo de las mujeres inmigrantes a los hombres inmigrantes, para comprobar si se observan los mismos patrones. También sería interesante analizar las pautas de fecundidad de matrimonios o parejas mixtas entre inmigrantes y españoles, o entre inmigrantes del mismo o de distinto origen (Cortina, Esteve y Domingo 2008). Otra línea de investigación de gran repercusión social es el contexto familiar de la fecundidad de los inmigrantes. Un estudio reciente (Cortina, Bueno y Castro Martín 2010) ha documentado que, a diferencia del nivel de fecundidad, en el que la distancia entre la población inmigrante y autóctona es pequeña o moderada, el comportamiento nupcial y el contexto conyugal de la reproducción después de la migración están fuertemente condicionados por los patro-

\footnotetext{
${ }^{20}$ En relación a esta cuestión, el análisis de biografías o event history analysis ha sido uno de los avances recientes más relevantes en las ciencias sociales (Blossfeld y Rohwer 2002).
} 
nes familiares y culturales imperantes en la sociedad de origen. En el caso del colectivo latinoamericano residente en España, por ejemplo, el $60 \%$ de los nacimientos ocurren en un contexto familiar no matrimonial. ${ }^{21}$ En qué medida esta estructura familiar puede mermar las oportunidades de las segundas generaciones es una cuestión a la que es importante dar respuesta.

\section{Referencias Bibliográficas}

Abbasi-Shavazi, M.J. y P. McDonald. 2002. "A comparison of fertility patterns of European immigrants in Australia with those in the countries of origin." Genus 58(1): 53-76.

Akee, R. 2010. "Who Leaves? Deciphering Immigrant Self-Selection from a Developing Country." Economic Development and Cultural Change 58(2): 323-344.

Álvarez Rodríguez, A. 2006. "Nacionalidad de los hijos de extranjeros nacidos en España". Madrid: Ministerio de Trabajo y Asuntos Sociales, Observatorio Permanente de la Inmigración. http:/l extranjeros.mtin.es/es/ObservatorioPermanentelnmigracion/Publicaciones/NacionalidadHijos. html. Consultado el 13 Enero 2010.

Andersson, G. 2004. "Childbearing after migration: Fertility patterns of foreign-born women in Sweden." International Migration Review 38(3): 747-774.

Arango, J. 2004. "La inmigración en España a comienzos del siglo XXI". Pp. 161-186 en Leal Maldonado, J. (ed.) Informe sobre la situación demográfica en España. Madrid: Fundación Fernando Abril Martorell.

Bledsoe, C. H. 2004. "Reproduction at the margins: Migration and legitimacy in the New Europe." Demographic Research: Special Collection 3, Article 4: 87-116. http://www.demographic-research.org/ special/3/4/. Consultado el 10 Enero 2010.

Bledsoe, C. H., R. Houle y P. Sow. 2007. "High fertility Gambians in low fertility Spain: The dynamics of child accumulation across transnational space." Demographic Research 16-12: 375-412. http:// www.demographic-research.org/Volumes/Vol16/12/default.htm. Consultado el 14 diciembre 2009.

Blossfeld, H-P. y G. Rohwer. 2002. Techniques of Event History Modeling: New Approaches to Causal Analysis. Hillsdale (NJ): Lawrence Erlbaum.

Castro Martín, T. 2002. "Consensual unions in Latin America: Persistence of a dual nuptiality system." Journal of Comparative Family Studies 33 (1): 35-55.

${ }^{21}$ En 2007, el 42,6\% de los nacimientos en España de mujeres latinoamericanas correspondió a madres en pareja de hecho y $16,9 \%$ a madres sin unión conyugal. Estos patrones se asemejan a los observados en América Latina, donde la prevalencia de uniones consensuales es elevada y donde el contexto legal de la unión conyugal no condiciona de forma relevante el comportamiento reproductivo (Castro Martín 2002). 
Castro Martín, T. y F. Juárez. 1995. "The Impact of Women's Education on Fertility in Latin America: Searching for Explanations." International Family Planning Perspectives 21(2): 52-57.

Cebolla, H. y A. González-Férrer. 2008. La inmigración en España (2000-2007). De la gestión de flujos a la integración de los inmigrantes. Madrid: Centro de Estudios Políticos y Constitucionales, Cuadernos y Debates, Vol. 184.

Cortina, C., X. Bueno García y T. Castro Martín. 2010. “¿Modelos familiares de aquí o de allá? Pautas de cohabitación entre las mujeres latinoamericanas en España." América Latina Hoy 55: 61-84.

Cortina, C., A. Esteve y A. Domingo. 2008. "Marriage Patterns of Foreign-Born Population in a New Country of Immigration: the Case of Spain." International Migration Review 42(4): 877-902.

Devolder, D. y R. Treviño. 2007. "Efectos de la inmigración extranjera sobre la evolución de la natalidad y de la fecundidad en España." Barcelona: Centre d'Estudis Demogràfics, Papers de Demografia ${ }^{\circ}$ 321. http://www.ced.uab.es/publicacions/PapersPDF/Text321.pdf. Consultado el 13 Enero 2010.

Díaz Gorfinkiel, M. 2008. "El Mercado de trabajo de los cuidados y la creación de las cadenas globales de cuidado: ¿cómo concilian las cuidadoras?." Cuadernos de Relaciones Laborales 26(2): 71-89.

Feliciano, C. 2005. "Educational selectivity in U.S. immigration: How do immigrants compare to those left behind?." Demography 42(1): 131-152.

Fernández Cordón, J. A. y C. Tobío Soler. 2005. Conciliar las responsabilidades familiares y laborales: políticas y prácticas sociales. Madrid: Fundación Alternativas, Documento de trabajo 79. http:/l www.falternativasrsc.org/files/docs/f7b6_09-03-06_792005.pdf. Consultado el 14 de Enero 2010.

Fokkema, T., H. De Valk, J. de Beer y C. Van Duin. 2008. "The Netherlands: Childbearing within the context of a "Poldermodel" society." Demographic Research 19-21: 743-794. http://www.demographicresearch.org/volumes/vol19/21/. Consultado el 20 Noviembre 2009.

Frank, R. y P. Heuveline. 2005. "A cross-over in Mexican and Mexican-American fertility rates: Evidence and explanations for an emerging paradox." Demographic Research 12- 4: 77-104. http://www. demographic-research.org/volumes/vol12/4/. Consultado el 21 Noviembre 2009.

Frejka, T. y T. Sobotka. 2008. "Fertility in Europe: Diverse, delayed and below replacement. Overview Chapter 1." T. Frejka et al. (eds.), Childbearing trends and policies in Europe. Demographic Research, Special Collection 7, 19-3: 15-46. http://www.demographic-research.org/Volumes/ Vol19/3/. Consultado el 13 Enero 2010.

Garssen, J. y H. Nicolaas. 2008. "Fertility of Turkish and Moroccan women in the Netherlands: Adjustment to native level within one generation". Demographic Research 19-33: 1249-1280. http://www. demographic-research.org/volumes/vol19/33/. Consultado el 20 Enero 2010.

Goldstein, J. R., T. Sobotka y A. Jasilioniene. 2009. "The End of 'Lowest-Low' Fertility? Max Planck Institute for Demographic Research", Working Paper WP 2009-029. http://www.demogr.mpg.de/ papers/working/wp-2009-029.pdf. Consultado el 25 Enero 2010.

González-Enríquez, C. 2009. Country report Spain. Report for the European Research ProjectCLANDESTINO. Undocumented Migration - Counting the Uncountable. Data and Trends across Europe. http://clandestino.eliamep.gr/clandestino-country-reports/Consultado el 10 Febrero 2010. 
Héran, F. y G. Pison. 2007. "Two children per woman in France in 2006: are immigrants to blame?." Population \& Societies 432: 1-4.

Hochschild, A. 2000. "Global Care Chains and Emotional Surplus Value". Pp. 130-146 en W. Hutton y A. Giddens (eds.) On the Edge: Living with Global Capitalism. London: Jonathan Cape.

Hoem, J.M. y L. Nedoluzhko. 2008. "Marriage formation as a process intermediary between migration and childbearing". Demographic Research 18-21: 611-628. http://www.demographic-research.org/ volumes/vol18/21/. Consultado el 22 Febrero 2010.

Hwang, S-S. y R. Saenz. 1997. "Fertility of Chinese immigrants in the U.S.: Testing a fertility emancipation hypothesis." Journal of Marriage and the Family 59(1): 50-61.

Instituto Nacional de Estadística (INE). 2009. "Encuesta Nacional de Inmigrantes 2007: una monografia". Madrid: INE. http://www.ine.es/prodyser/pubweb/eni07/eni07.htm. Consultado el 24 Noviembre 2009.

Izquierdo, A. y D. López de Lera (eds.). 2006. Demografía de los extranjeros: incidencia en el crecimiento de la población. Bilbao: Fundación Banco Bilbao Vizcaya Argentaria.

Kohler, H-P., F. C. Billari, y J. A. Ortega. 2002. "The emergence of lowest-low fertility in Europe during the 1990s." Population and Development Review 28(4): 641-681.

Kulu, H. 2005. "Migration and fertility: competing hypotheses re-examined". European Journal of Population 21(1): 51-87.

Lindstrom, D. P. y S. Giorguli Saucedo. 2007. "The interrelationship of fertility, family maintenance, and Mexico-U.S." Migration. Demographic Research 17-28: 821-858. http://www.demographicresearch.org/volumes/vol17/28/. Consultado el 17 Noviembre 2009.

Lorenzo, L., E. Suárez y T. Castro Martín. 2007. "Perfil laboral, familiar y residencial de los inmigrantes en Madrid a la luz del Censo de 2001”. Pp. 371-383 en Ascensión Calatrava et al.,. (Eds.), Economías, mercados de trabajo y territorios metropolitanos en transformación. Madrid: ARETHUSE.

Malmusi, D. y J. María Jansà. 2007. "Recomendaciones para la investigación e información en salud sobre definiciones y variables para el estudio de la población inmigrante de origen extranjero." Revista Española de Salud Pública 81(004): 399-409.

Mamolo, M. y S. Scherbov. 2009. "Population Projections for Forty-Four European Countries: The Ongoing Population Ageing". European Demographic Research Papers 2, Vienna Institute of Demography, http://www.oeaw.ac.at/vid/download/edrp_2_09.pdf. Consultado el 14 Enero 2010.

Martínez Buján, R. 2008. "Los beneficios de la inmigración al Estado de Bienestar. Mujeres inmigrantes en el sector doméstico de cuidados". Capítulo 7 del VI Informe FOESSA sobre Exclusión y Desarrollo Social en España 2008. Madrid: Fundación FOESSA y Cáritas Española Editores.

Mayer, J. y R. T. Riphahn. 2000. "Fertility Assimilation of Immigrants: Evidence from Count Data Models." Journal of Population Economics 13(2): 241-261.

McDonald, P. 2000. "Gender equity, social institutions and the future of fertility." Journal of Population Research 17(1): 1-16. 
Milewski, N. 2007. "First child of immigrant workers and their descendants in West Germany: Interrelation of events, disruption, or adaptation?" Demographic Research 17-29: 859-896. http://www. demographic-research.org/Volumes/Vol17/29/. Consultado el 11 Diciembre 2009.

Nahmias, P. 2004. "Fertility behavior of recent immigrants to Israel: a comparative analysis of immigrants from Ethiopia and the former Soviet Union. Demographic Research 10-4: 83-120. http:// www.demographic-research.org/Volumes/Vol10/4/. Consultado el 24 Septiembre 2009.

Navarro, V. 2006. El subdesarrollo social de España: causas y consecuencias. Barcelona: Anagrama.

Ortega, J. A. y H.-P. Kohler. 2001. "¿Está cayendo realmente la fecundidad española?: Separación de los efectos intensidad, calendario y varianza en el Índice Sintético de Fecundidad." Revista Española de Investigaciones Sociológicas 96: 95-122.

Parrado, E. 2011. "How high is Hispanic/Mexican fertility in the U.S.? Immigration and tempo considerations. Demography (en prensa).

Reher, D. y M. Requena. 2009a. "The National Immigrant Survey of Spain: A new data source for migration studies in Europe." Demographic Research 20-12: 253-278. http://www.demographic-research. org/Volumes/Vol20/12/20-12.pdf. Consultado el 12 Enero 2010.

Reher, D. y M. Requena (eds.). 2009b. Las múltiples caras de la inmigración en España. Madrid: Alianza Editorial.

Rodríguez, G. y J. Cleland. 1988. "Modelling Marital Fertility by Age and Duration: An Empirical Appraisal of the Page Model." Population Studies 42(2): 241-257.

Roig, M. y T. Castro Martín. 2007. "Childbearing Patterns of Foreign Women in a New Immigration Country: The Case of Spain." Population English edition 62(3): 351-380; Population Édition française 62(3): 419-446.

Rosero-Bixby, L., T. Castro Martín y T. Martín García. 2009. "Is Latin America starting to retreat from early and universal childbearing?." Demographic Research 20-9: 169-194. http://www.demographic-research.org/Volumes/Vol20/9/20-9.pdf.

Rosero-Bixby, L., T. Castro Martín, D. Reher y M. Sánchez. 2009. "How many immigrants live in Spain? An alternative estimation to the population register based on births and fertility rates". IUSSP International Population Conference, Marrakech, 27 Septiembre - 2 Octubre 2009.

Sardon, J-P. 2006. "Recent demographic trends in the developed countries." Population-E 61(3): 197266.

Sobotka, T. 2004. "Is lowest-low fertility explained by the postponement of childbearing?." Population and Development Review 30(2): 195-220.

Sobotka, T. 2008. "The rising importance of migrants for childbearing in Europe." Demographic Research 19(9): 225-248. http://www.demographic-research.org/Volumes/Vol19/9/. Consultado el 14 Enero 2010. 
Sobotka, T. y W. Lutz. 2009. "Misleading Policy Messages from the Period TFR: Should We Stop Using It?" European Demographic Research Papers 4, Vienna Institute of Demography. http://www.oeaw. ac.at/vid/download/edrp_4_09.pdf. Consultado el 15 Enero 2010.

Statcorp. 2009. Stata Statistical Software: release 10.1. College Station, Texas: Stata Corporation.

Tobío, C. y M. Díaz Gorfinkiel. 2007. "New gendered relationships in Spain: 'the other' in the care triangle". International Journal of Iberian Studies 20(1): 41-63.

Toulemon, L. 2004. "Fertility among immigrant women: new data, a new approach". Population \& Societies No. 400: 1-4. http://www.ined.fr/en/resources_documentation/publications/pop_soc/bdd/publication/540/. Consultado el 16 Enero 2010.

Toulemon, L. 2006. "Fertility Among Immigrant Women in France: New Data, a New Approach. Population Association of American 2006 Annual Meeting, Los Angeles, 30 Marzo-1 Abril 2006. http:/l paa2006. princeton.edu/download.aspx?submissionld=61103. Consultado el 16 Enero 2010.

Toulemon, L. y M. Mazuy. 2004. "Comment prendre en compte l'âge à l'arrivée et la durée de séjour en France dans la mesure de la fécondité des immigrants?" París: INED, Document de travail 120. http://www.ined.fr/fichier/t_publication/1053/publi_pdf1_120.pdf. Consultado el 18 Enero 2010.

Wagner, H. 2008. "Maternidad transnacional: discursos, estereotipos, prácticas (2008)" en G. Herrera y J. Ramírez (eds.), América Latina migrante: Estado, familia, identidades. Quito: FLACSO y Ministerio de Cultura del Ecuador.

TERESA CASTRO MARTíN es Doctora en Sociología por la Universidad de Wisconsin-Madison (1990). De 1990 a 1996, trabajó en la División de Población de Naciones Unidas y en 1996 se incorporó al CSIC, donde actualmente es Profesora de Investigación. Sus investigaciones se centran en los procesos de cambio de las dinámicas familiares, en las interacciones entre biografías conyugales, reproductivas y laborales de los jóvenes, y en temas de salud sexual y reproductiva desde de una perspectiva internacional.

LUIS ROSERO BIXBY es Catedrático de la Universidad de Costa Rica y fundador y ex director del Centro Centroamericano de Población en esa universidad. Es PhD en Población y Magister en Salud Pública por la Universidad de Michigan y Economista por la Pontificia Universidad Católica del Ecuador. Es miembro de la Academia Nacional de Ciencias de Costa Rica y del Grupo de Estudios 'Población y Sociedad'.

\section{RECIBIDO: 17/11/2010}

ACEPTADO: 01/02/2011 